\title{
Introduction to disaggregate demand models
}

\author{
Michel Bierlaire
}

Transport and Mobility Laboratory

School of Architecture, Civil and Environmental Engineering

Ecole Polytechnique Fédérale de Lausanne

January 28, 2013 


\section{Outline}

(1) Demand models

(2) Choice theory

(3) Operational model

(4) Market shares

(5) Willingness to pay

(6) Price optimization

(7) Summary

$\checkmark$ TRANSP-OR

\section{(PPA}

ECOLI POLYTECHNIQUE FEDIRALE DE LAUSANNE 


\section{Demand models}

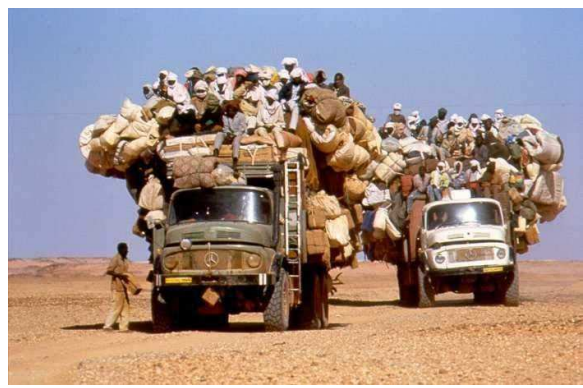

- Supply = infrastructure

- Demand $=$ behavior, choices

- Congestion $=$ mismatch 


\section{Demand models}

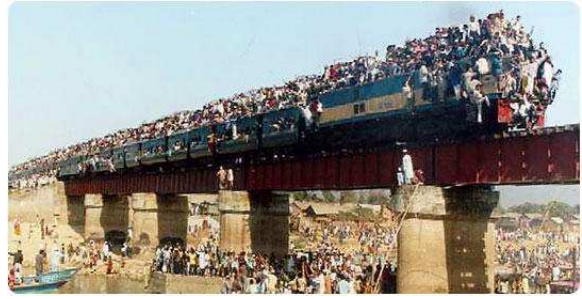

- Usually in OR:

- optimization of the supply

- for a given (fixed) demand

\section{(PPA}

ICOLI POLYTICHNIQUE EDIRALE DE LAUSANNE 


\section{Aggregate demand}

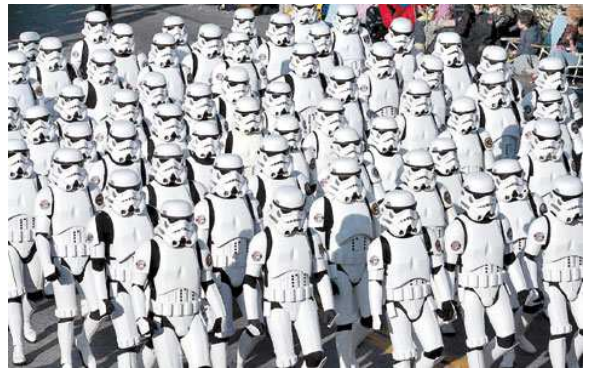

- Homogeneous population

- Identical behavior

- Price $(P)$ and quantity $(Q)$

- Demand functions: $P=f(Q)$

- Inverse demand: $Q=f^{-1}(P)$ 


\section{Disaggregate demand}

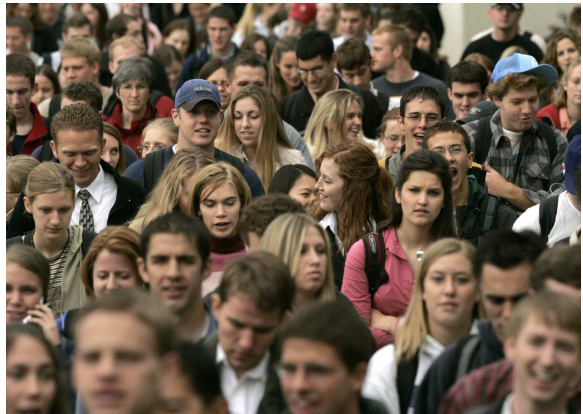

- Heterogeneous population

- Different behaviors

- Many variables:

- Attributes: price, travel time, reliability, frequency, etc.

- Characteristics: age, income, education, etc.

- Complex demand/inverse demand functions. 


\section{(2) Choice theory}

(3) Operational model

(4) Market shares

(5) Willingness to pay

(6) Price optimization

(1) Summary

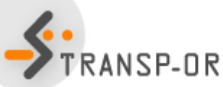

\section{(PFI}

EOOLI POLYTECHNIQUE FDIRALE DE LAUSANNE

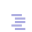

M. Bierlaire (TRANSP-OR ENAC EPFL) Introduction to disaggregate demand models
January 28, 2013

$7 / 58$ 


\section{Choice}

Choice: outcome of a sequential decision-making process

- Definition of the choice problem: How do I get to work?

- Generation of alternatives: car as driver, car as passenger, train

- Evaluation of the attributes of the alternatives: price, time, flexibility, comfort

- Choice: decision rule

- Implementation: travel 


\section{Choice theory}

A choice theory defines

(3) decision maker

(2) alternatives

(3) attributes of alternatives

(3) decision rule 


\section{Decision-maker}

The decision maker is

- an individual or a group of persons.

- If group of persons, we ignore internal interactions.

- Important to capture difference in tastes and decision-making processes.

- Socio-economic characteristics: age, gender, income, education, etc. 


\section{Alternatives}

Choice set

- Environment: universal choice $\operatorname{set}(\mathcal{U})$

- Individual $n$ : choice set $\left(\mathcal{C}_{n}\right)$

Choice set generation

- Availability

- Awareness

Choice set type

- Continuous

- Discrete 


\section{Continuous vs. discrete}

Continuous choice set

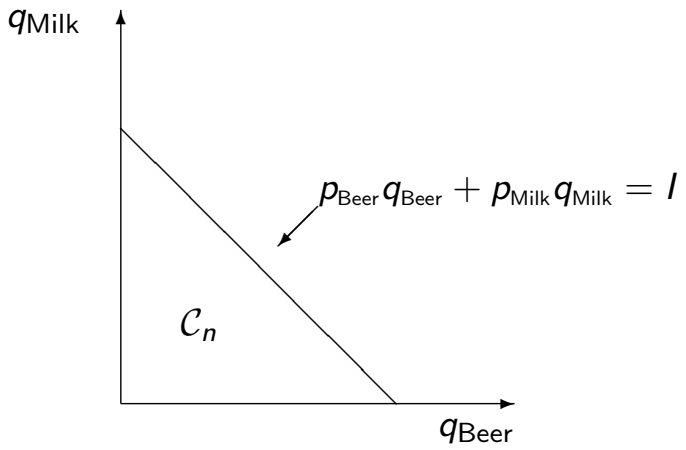

Discrete choice set

$$
\mathcal{C}_{n}=\{\text { Car, Bus, Bike }\}
$$




\section{Attributes}

Describe the item:

- cost

- travel time

- walking time

- comfort

- bus frequency

- etc. 


\section{Decision rules}

Neoclassical economic theory

Preference-indifference operator $\gtrsim$

(1) reflexivity

$$
a \gtrsim a \quad \forall a \in \mathcal{C}_{n}
$$

(2) transitivity

$$
a \gtrsim b \text { and } b \gtrsim c \Rightarrow a \gtrsim c \quad \forall a, b, c \in \mathcal{C}_{n}
$$

(3) comparability

$$
a \gtrsim b \text { or } b \gtrsim a \quad \forall a, b \in \mathcal{C}_{n}
$$




\section{Decision rules}

Utility

$$
\begin{gathered}
\exists U_{n}: \mathcal{C}_{n} \longrightarrow \mathbb{R}: a \rightsquigarrow U_{n}(a) \text { such that } \\
a \gtrsim b \Leftrightarrow U_{n}(a) \geq U_{n}(b) \quad \forall a, b \in \mathcal{C}_{n}
\end{gathered}
$$

Remarks

- Utility is a latent concept

- It cannot be directly observed 


\section{Utility and continuous choice set}

Context for the decision maker

- $Q=\left\{q_{1}, \ldots, q_{L}\right\}$ consumption bundle

- $q_{i}$ is the quantity of product $i$ consumed

- Utility of the bundle:

$$
U\left(q_{1}, \ldots, q_{L}\right)
$$

- $Q_{a} \gtrsim Q_{b}$ iff $U\left(q_{1}^{a}, \ldots, q_{L}^{a}\right) \geq U\left(q_{1}^{b}, \ldots, q_{L}^{b}\right)$

- Budget constraint:

$$
\sum_{i=1}^{L} p_{i} q_{i} \leq l .
$$




\section{Utility and continuous choice set}

Decision-maker solves the optimization problem

$$
\max _{q \in \mathbb{R}^{L}} U\left(q_{1}, \ldots, q_{L}\right)
$$

subject to

$$
\sum_{i=1}^{L} p_{i} q_{i}=l
$$

Example with two products

$$
\max _{q_{1}, q_{2}} U=\beta_{0} q_{1}^{\beta_{1}} q_{2}^{\beta_{2}}
$$

subject to

$$
p_{1} q_{1}+p_{2} q_{2}=I \text {. }
$$




\section{Utility and continuous choice set}

Example with two products

$$
\max _{q_{1}, q_{2}} U=\beta_{0} q_{1}^{\beta_{1}} q_{2}^{\beta_{2}}
$$

subject to

$$
p_{1} q_{1}+p_{2} q_{2}=I .
$$

Lagrangian of the problem

$$
L\left(q_{1}, q_{2}, \lambda\right)=\beta_{0} q_{1}^{\beta_{1}} q_{2}^{\beta_{2}}+\lambda\left(I-p_{1} q_{1}-p_{2} q_{2}\right) .
$$

Necessary optimality condition (KKT)

$$
\nabla L\left(q_{1}, q_{2}, \lambda\right)=0
$$




\section{Utility and continuous choice set}

KKT

$$
\begin{aligned}
\beta_{0} \beta_{1} q_{1}^{\beta_{1}-1} q_{2}^{\beta_{2}}-\lambda p_{1} & =0 \\
\beta_{0} \beta_{2} q_{1}^{\beta_{1}} q_{2}^{\beta_{2}-1}-\lambda p_{2} & =0 \\
p_{1} q_{1}+p_{2} q_{2}-I & =0 .
\end{aligned}
$$

We have

$$
\begin{aligned}
& \beta_{0} \beta_{1} q_{1}^{\beta_{1}} q_{2}^{\beta_{2}}-\lambda p_{1} q_{1}=0 \\
& \beta_{0} \beta_{2} q_{1}^{\beta_{1}} q_{2}^{\beta_{2}}-\lambda p_{2} q_{2}=0
\end{aligned}
$$

so that

$$
\lambda I=\beta_{0} q_{1}^{\beta_{1}} q_{2}^{\beta_{2}}\left(\beta_{1}+\beta_{2}\right)
$$

\section{KKT (ctd.)}

Therefore

$$
\beta_{0} q_{1}^{\beta_{1}} q_{2}^{\beta_{2}}=\frac{\lambda l}{\left(\beta_{1}+\beta_{2}\right)}
$$

As $\beta_{0} \beta_{2} q_{1}^{\beta_{1}} q_{2}^{\beta_{2}}=\lambda p_{2} q_{2}$, we obtain (assuming $\lambda \neq 0$ )

$$
q_{2}=\frac{I \beta_{2}}{p_{2}\left(\beta_{1}+\beta_{2}\right)}
$$

Similarly, we obtain

$$
q_{1}=\frac{I \beta_{1}}{p_{1}\left(\beta_{1}+\beta_{2}\right)}
$$




\section{Utility and continuous choice set}

Demand functions

$$
\begin{aligned}
q_{1} & =\frac{I \beta_{1}}{p_{1}\left(\beta_{1}+\beta_{2}\right)} \\
q_{2} & =\frac{I \beta_{2}}{p_{2}\left(\beta_{1}+\beta_{2}\right)}
\end{aligned}
$$




\section{Utility and discrete choice set}

Binary optimization

$$
\max _{q \in\{0,1\}^{L}} U=U\left(q_{1}, \ldots, q_{L}\right)
$$

with

$$
q_{i}= \begin{cases}1 & \text { if product } i \text { is chosen } \\ 0 & \text { otherwise }\end{cases}
$$

and

$$
\sum_{i} q_{i}=1
$$

No optimality condition. Calculus cannot be used anymore. 


\section{Methodology}

Utility functions

- Do not work with demand functions anymore

- Work with utility functions

- $U$ is the "global" utility

- Define $U_{i}$ the utility associated with product $i$.

- It is a function of the attributes of the product (price, quality, etc.)

- We say that product $i$ is chosen if

$$
U_{i} \geq U_{j} \quad \forall j
$$




\section{Example}

Two transportation modes

$$
\begin{aligned}
& U_{1}=-\beta t_{1}-\gamma c_{1} \\
& U_{2}=-\beta t_{2}-\gamma c_{2}
\end{aligned}
$$

with $\beta, \gamma>0$

$$
U_{1} \geq U_{2} \text { iff }-\beta t_{1}-\gamma c_{1} \geq-\beta t_{2}-\gamma c_{2}
$$

that is

$$
-\frac{\beta}{\gamma} t_{1}-c_{1} \geq-\frac{\beta}{\gamma} t_{2}-c_{2}
$$

or

$$
c_{1}-c_{2} \leq-\frac{\beta}{\gamma}\left(t_{1}-t_{2}\right)
$$




\section{Example}

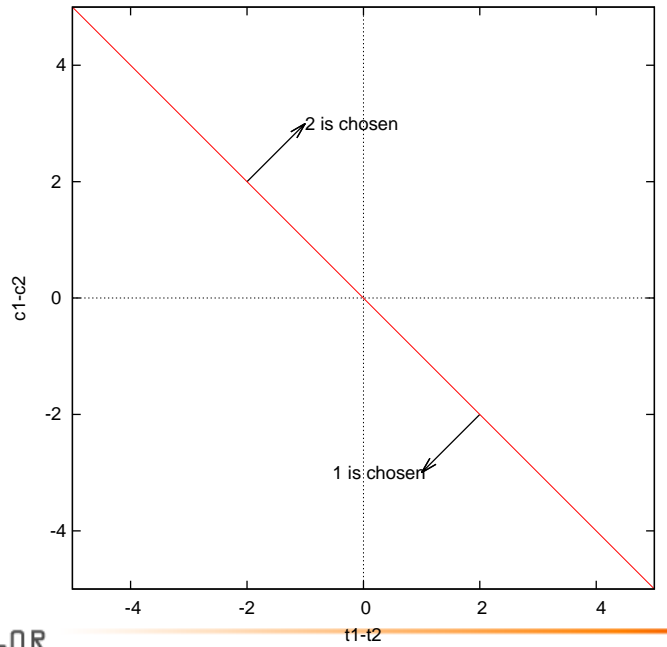

\section{(PPF}

EOOLI POLYTECHNIQUE FEDIRMLE DE LAUSANNE

를 


\section{Example}

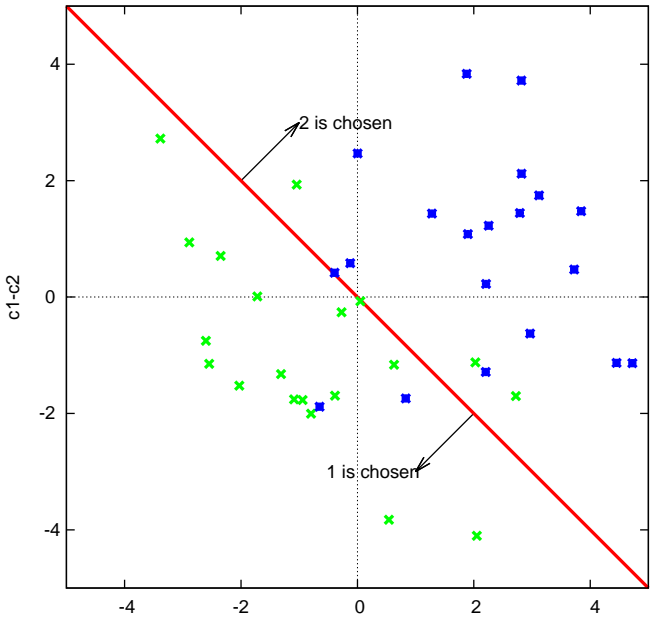

\section{(fPf}

EOLI POLYTECHNIQUE EDIRALE DE LAUSANNE 


\section{Assumptions}

Decision-maker

- perfect discriminating capability

- full rationality

- permanent consistency
Analyst

- knowledge of all attributes

- perfect knowledge of $\gtrsim$ (or $\left.U_{n}(\cdot)\right)$

- no measurement error

Must deal with uncertainty

- Random utility models

- For each individual $n$ and alternative $i$

$$
U_{i n}=V_{i n}+\varepsilon_{i n}
$$

and

$$
P\left(i \mid \mathcal{C}_{n}\right)=P\left[U_{i n}=\max _{j \in \mathcal{C}_{n}} U_{j n}\right]=P\left(U_{i n} \geq U_{j n} \forall j \in \mathcal{C}_{n}\right)
$$




\section{Daniel L. McFadden}

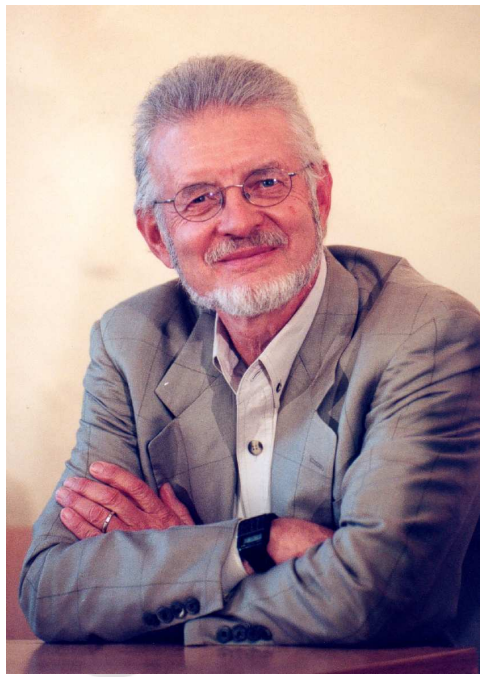

- UC Berkeley 1963, MIT 1977, UC Berkeley 1991

- Laureate of The Bank of Sweden Prize in Economic Sciences in Memory of Alfred Nobel 2000

- Owns a farm and vineyard in Napa Valley

- "Farm work clears the mind, and the vineyard is a great place to prove theorems" 


\section{(1) Demand models}

\section{(2) Choice theory}

\section{(3) Operational model}

(4) Market shares

(5) Willingness to pay

(6) Price optimization

(7) Summary

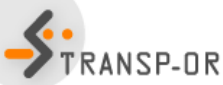

\section{(APF}

ECOLI POLYTECHNIQUE FEDIRALE DE LAUSANNE

플

M. Bierlaire (TRANSP-OR ENAC EPFL) Introduction to disaggregate demand models
January 28, 2013

$28 / 58$ 


\section{Assumptions on $V_{\text {in }}$}

$$
V_{i n}=V\left(x_{i n}\right)=V\left(z_{i n}, S_{n}\right)
$$

Variables

- $z_{i n}$ : vector of attributes of alternative $i$ for individual $n$

- $S_{n}$ : vector of socio-economic characteristics of $n$

- $x_{i n}=\left(z_{i n}, S_{n}\right)$

Functional form

- Common assumption: Linear-in-parameter

$$
V_{\text {in }}=\sum_{p} \beta_{p}\left(x_{i n}\right)_{p}
$$

- $\beta$ : vector of unknown parameters to be estimated

- Possibility for nonlinear specifications. 


\section{Assumptions on $\varepsilon_{\text {in }}$}

- Mean: parameter to be estimated.

- Variance: parameter that cannot be estimated, must be normalized.

- Distribution: two common assumptions

Probit model

- Normal distribution

- Motivation: central limit theorem

TRANSP-OR
Logit model

- Extreme value distribution

- i.i.d. across $i$ and $n$

- Motivation: Gumbel's theorem 


\section{Logit model}

Utility

$$
U_{i n}=V_{i n}+\varepsilon_{i n}
$$

Choice probability

$$
P_{n}\left(i \mid \mathcal{C}_{n}\right)=\frac{e^{V_{i n}}}{\sum_{j \in \mathcal{C}_{n}} e^{V_{j n}}} .
$$

- Decision-maker $n$

- Alternative $i \in \mathcal{C}_{n}$ 


\section{Model estimation}

- Design a sampling protocol

- For each individual $n$ in the sample, and for each alternative $i$,

- observe the explanatory variables $x_{i n}$

- observe the dependent variable, that is the choice $i_{n}$.

- Compute the likelihood as a function of the parameters $\beta$ :

$$
\Lambda_{n}(\beta)=P_{n}\left(i_{n} \mid C_{n}\right)=\frac{e^{V_{i_{n} n}}}{\sum_{j \in \mathcal{C}_{n}} e^{V_{j n}} .}
$$

- Compute the likelihood of the whole sample:

$$
\mathcal{L}^{*}(\beta)=\prod_{n} \Lambda_{n}(\beta)
$$

- Solve the maximum likelihood problem:

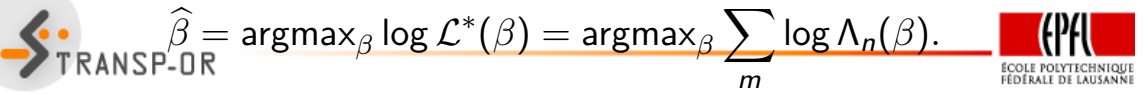




\section{Demand models}

Choice theory

Operational model

(4) Market shares

(5) Willingness to pay

(6) Price optimization

(7) Summary

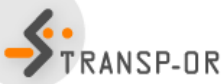




\section{Aggregation}

\section{Reality}

- Population composed of $N$ individuals

- For each alternative $i$, and each individual $n$, define

$$
y_{i n}= \begin{cases}1 & \text { if individual } n \text { chooses alternative } i \\ 0 & \text { otherwise }\end{cases}
$$

- Total number of individuals selecting item $i$ in the population:

$$
N(i)=\sum_{n=1}^{N} y_{i n}
$$

- Market share for item $i$ :

$$
W(i)=N(i) / N=\sum_{n=1}^{N} y_{i n} / N .
$$




\section{Forecasting}

- Replace $y_{i n}$ by $P_{n}\left(i \mid x_{n}, C_{n}\right)$

- Total number of individuals selecting item $i$ in the population:

$$
\widehat{N}(i)=\sum_{n=1}^{N} P_{n}\left(i \mid x_{n}, C_{n}\right)
$$

- Market share for item $i$ :

$$
\widehat{W}(i)=\widehat{N}(i) / N=\sum_{n=1}^{N} P_{n}\left(i \mid x_{n}, C_{n}\right) / N .
$$

- Issue: no way to access $x_{n}$ for the entire population.

- Solution: use a representative sample $\rightarrow$ TRANSP-OR 


\section{Demand models}

- $\widehat{N}(i)$ or $\widehat{W}(i)$ are the demand models required for OR

- They can be computed for subgroups of the population

- They account for the heterogeneity of

- behavior,

- taste,

- choice contexts. 


\section{(1) Demand models}

(2) Choice theory

(3) Operational model

(4) Market shares

\section{(5) Willingness to pay}

(6) Price optimization

(7) Summary

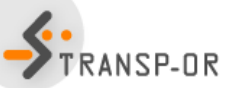

\section{(PFI}

ICOLI POLYTICHNIQUE FEDIRALE DE LAUSANNE 플

M. Bierlaire (TRANSP-OR ENAC EPFL) Introduction to disaggregate demand models
January 28, 2013

$37 / 58$ 


\section{Value of time}

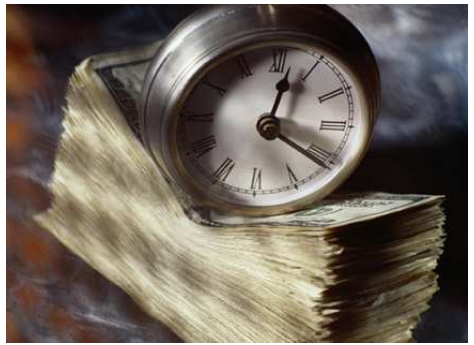

Objective

- Monetary value of travel time?

- Cost - benefit analysis

- Costs : CHF

- Benefits : travel time savings

Definition

Price that travelers are willing to pay to decrease the travel time.

Motivation

Total time budget is limited, saved time can be used for other activities and, therefore, has value. 


\section{Example}

Utility functions

$$
\begin{aligned}
& U_{1}=-\beta t_{1}-\gamma c_{1} \\
& U_{2}=-\beta t_{2}-\gamma c_{2}
\end{aligned}
$$

with $\beta, \gamma>0$

Choice assumption

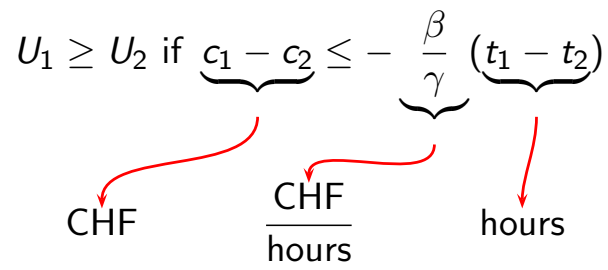




\section{Value of time}

- If utility function is linear

- the value of time is the ratio between

- the coefficient of the "time" variable, and

- the coefficient of the "cost" variable

- Warning: utility is not always linear

- Value of time varies with

- trip purpose

- transportation mode

- trip length

- income 


\section{Example: model choice in Nijmegen}

$$
\begin{array}{rll}
V_{\text {car }}=-0.798 & -0.110 \cdot \text { cost }_{\text {car }} & -1.33 \cdot \text { time }_{\text {car }} \\
V_{\text {train }}= & -0.110 \cdot \text { cost }_{\text {train }} & -1.33 \cdot \text { time }_{\text {train }}
\end{array}
$$

Value of time $=-1.33 /-0.110 \approx 12$ euros $/ \mathrm{h} \approx 0.20$ euros $/ \mathrm{min}$

\begin{tabular}{lll} 
& Case 1 & Case 2 \\
\hline Time & $2 \mathrm{~h}$ & $1.5 \mathrm{~h}$ \\
Cost & $7 €$ & $13 €$ \\
Utility of train & -3.43 & -3.43
\end{tabular}




\section{Other willingness to pay indicators}

- Headway (i.e. time between two buses)

- Number of transfers

- Reliability

- etc.

Same methodology:

- The model must involve the corresponding variable

- Willingness-to-pay = ratio between the coefficient of the variable and the cost coefficient

$$
U=-\beta t-\gamma c-\alpha n
$$

Willingness-to-pay to have one less transfer: $\alpha / \gamma$ 


\section{Value of time in Switzerland}

\section{Reference}

Axhausen, K., Hess, S., Koenig, A., Abay, G., Bates, J., and Bierlaire, M. (2008). Income and distance elasticities of values of travel time savings: new Swiss results, Transport Policy 15(3):173-185.

Data collection

- Source for recruitment: survey "Kontinuierliche Erhebung zum Personenverkehr" (KEP) by SBB/CFF

- Stated preferences

- Questionnaire designed based on a real reference trip

- Three parts:

- SP mode choice (car / bus or rail)

- SP route choice (current mode or alternative mode)

- Socio-demographics and information about the reference trip 


\section{SP survey}

Mode choice car - rail (main study version)

\begin{tabular}{ll|}
\hline Travel costs: & $18 \mathrm{Fr}$. \\
\hline Total travel time: & 40 minutes \\
\hline ... congested: & 10 minutes \\
\hline ... uncongested: & 30 minutes \\
\hline
\end{tabular}

\begin{tabular}{|l|l|}
\hline & \\
\hline Travel costs: & $23 \mathrm{Fr}$. \\
\hline Travel time: & 30 minutes \\
\hline Headway: & 30 minutes \\
\hline No. of changes: & 0 times \\
\hline
\end{tabular}

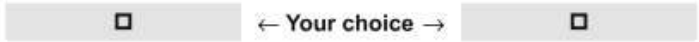

Route choice rail (main study version)

\begin{tabular}{|ll|}
\hline Travel costs: & $20 \mathrm{Fr}$. \\
\hline Travel time: & 40 minutes \\
\hline Headway: & 15 minutes \\
\hline No. of changes: & 1 times \\
\hline
\end{tabular}

\begin{tabular}{|l|l|}
\hline & \\
\hline Travel costs: & $23 \mathrm{Fr}$. \\
\hline Travel time: & 30 minutes \\
\hline Headway: & 30 minutes \\
\hline No. of changes: & 0 times \\
\hline
\end{tabular}
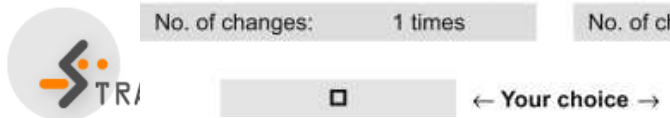

ㅁ

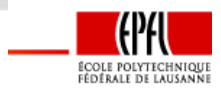




\section{Data}

Number of observations (1225 individuals)

\begin{tabular}{l|rrrr|r} 
& Busin. & Comm. & Leis. & Shop. & Total \\
\hline Mode : car/bus & 6 & 162 & 186 & 126 & 480 \\
Mode : car/rail & 426 & 1716 & 2538 & 1104 & 5784 \\
Route : bus for bus users & 9 & 405 & 450 & 342 & 1206 \\
Route : car for car users & 156 & 846 & 1176 & 660 & 2838 \\
Route : rail for car users & 126 & 594 & 837 & 504 & 2061 \\
Route : rail for rail users & 324 & 1008 & 1881 & 288 & 3501 \\
\hline Total & 1047 & 4731 & 7068 & 3024 & 15870
\end{tabular}

In average, $15870 / 1225 \approx 13$ valid responses out of 16 per respondent. 


\section{Model specification}

Variables
- travel time
- inertia
- travel cost
- car availability
- level of congestion (car)
- sex
- frequency (TC)
- 1/2-fare CFF
- number of transfers (TC)
- general subscription
- trip length
- trip purpose
- income 


\section{Results}

\begin{tabular}{l|rrrr} 
& Business & Commute & Leisure & Shopping \\
\hline Time TC $(\mathrm{CHF} / \mathrm{h})$ & 49.57 & 27.81 & 21.84 & 17.73 \\
Time car $(\mathrm{CHF} / \mathrm{h})$ & 50.23 & 30.64 & 29.20 & 24.32 \\
Headway $(\mathrm{CHF} / \mathrm{h})$ & 14.88 & 11.18 & 13.38 & 8.48 \\
CHF/transfer & 7.85 & 4.89 & 7.32 & 3.52
\end{tabular}




\section{Results}
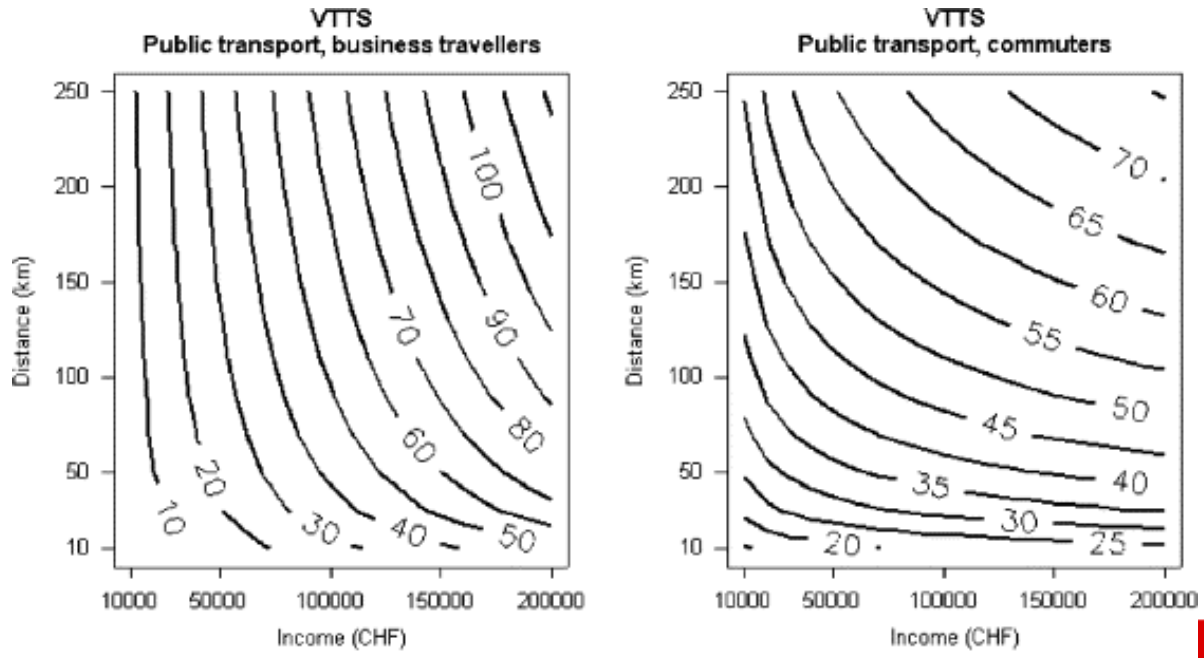


\section{Demand models}

Choice theory

Operational model

Market shares

(5) Willingness to pay

(6) Price optimization

(7) Summary

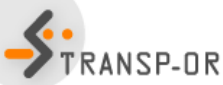

\section{(PFI}

ECOLI POLYTECHNIQUE FETRMLE DE LAUSANNE

플

M. Bierlaire (TRANSP-OR ENAC EPFL) Introduction to disaggregate demand models
January 28, 2013

$49 / 58$ 


\section{Introduction}

- Choice model captures demand

- Demand is elastic to price

- Predicted demand varies with price, if it is a variable of the model

- In principle, the probability to use/purchase an alternative decreases if the price increases.

- The revenue per user increases if the price increases.

- Question: what is the optimal price to optimize revenue?

In short

- Price $\uparrow \Rightarrow$ profit/passenger $\uparrow$ and number of passengers $\downarrow$

- Price $\downarrow \Rightarrow$ profit/passenger $\downarrow$ and number of passengers $\uparrow$

- What is the best trade-off? 


\section{Revenue calculation}

Number of persons choosing alternative $i$ in the population

$$
\hat{N}(i)=\sum_{s=1}^{S} N_{s} P\left(i \mid x_{s}, p_{i s}\right)
$$

where

- the population is segmented into $S$ homogeneous strata

- $p_{i s}$ is the price of item $i$ in segment $s$

- $x_{s}$ gathers all other variables corresponding to segment $s$

- $P\left(i \mid x_{s}, p_{i s}\right)$ is the choice model

- $N_{s}$ is the number of individuals in segment $s$ 


\section{Revenue calculation}

Total revenue from $i$

$$
R_{i}=\sum_{s=1}^{S} N_{s} P\left(i \mid x_{s}, p_{i s}\right) p_{i s}
$$

If the price is constant across segments...

$$
R_{i}=p_{i} \sum_{s=1}^{S} N_{s} P\left(i \mid x_{s}, p_{i}\right)
$$




\section{Price optimization}

Optimizing the price of product $i$ is solving the problem

$$
\max _{p_{i}} p_{i} \sum_{s=1}^{S} N_{s} P\left(i \mid x_{s}, p_{i}\right)
$$

Notes:

- It assumes that everything else is equal

- In practice, it is likely that the competition will also adjust the prices 


\section{Illustrative example}

A binary logit model with

$$
\begin{aligned}
& V_{1}=\beta_{p} p_{1}-0.5 \\
& V_{2}=\beta_{p} p_{2}
\end{aligned}
$$

so that

$$
P(1 \mid p)=\frac{e^{\beta_{p} p_{1}-0.5}}{e^{\beta_{p} p_{1}-0.5}+e^{\beta_{p} p_{2}}}
$$

Two groups in the population:

- Group 1: $\beta_{p}=-2, N_{s}=600$

- Group 2: $\beta_{p}=-0.1, N_{s}=400$

Assume that $p_{2}=2$. 


\section{Illustrative example}

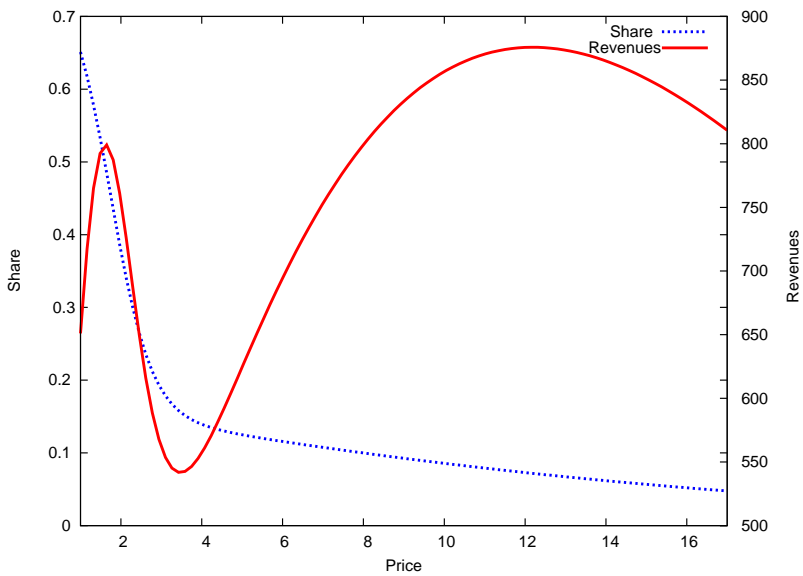




\section{Sensitivity analysis}

- Parameters are estimated, we do not know the real value

- 95\% confidence interval: $\left[\widehat{\beta}_{p}-1.96 \sigma, \widehat{\beta}_{p}+1.96 \sigma\right]$

- Perform a sensitivity analysis for $\beta_{p}$ in group 2 


\section{Sensitivity analysis}

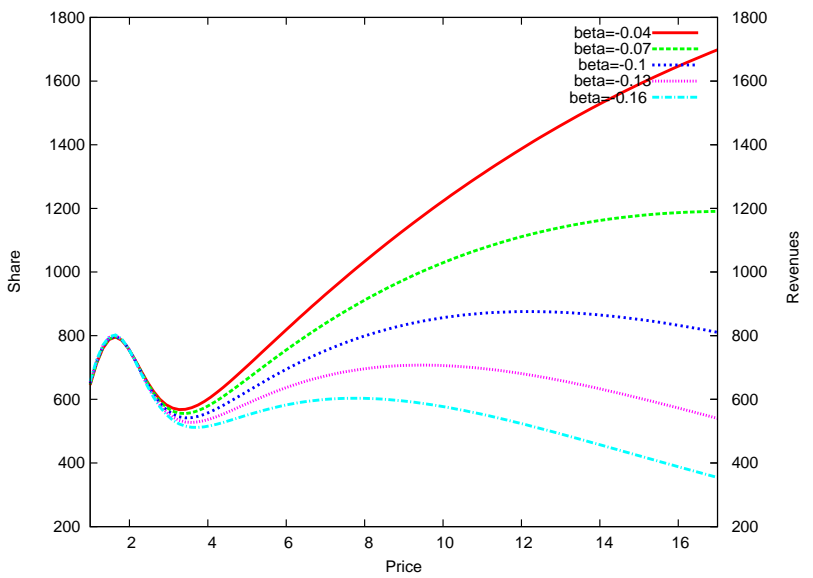




\section{Summary}

(1) Demand models

(2) Choice theory

(3) Operational model

(4) Market shares

(5) Willingness to pay

(6) Price optimization

(7) Summary 